\title{
Historia del clúster de calzado en Jalisco y su impacto en la estructura industrial
}

\author{
CARLOS EDUARDO LÓPEZ-HERNÁNDEZ ${ }^{a}$
}

RESUMEN La historia de la industria del calzado en México demuestra que su declive obedece a muchas variables, como, por ejemplo, la apertura de los mercados internacionales y los costos de mano de obra, pero también la estructura organizacional de tipo industrial como lo es el fortalecimiento del clúster. En México, al igual que otros países como Estados Unidos, Inglaterra, China y Brasil, la industria del calzado se localiza y concentra en algunas regiones geográficas, de manera que el fortalecimiento de las relaciones existentes entre los participantes del clúster es lo que desarrolla y hace crecer la industria. En este trabajo de investigación se describe la estructura industrial del clúster de calzado en Jalisco y las transformaciones históricas que ha sufrido.

PALABRAS CLAVE clúster, calzado, grandes empresas y crecimiento.

\section{HISTORIA DEL ARTÍCULO}

¿CÓMO CITAR?:

López-Hernández, C.E. (2018).

Historia del clúster de calzado en Jalisco y su impacto en la estructura industrial. Perspectiva Empresarial, 4(2), 69-77. http://dx.doi. org/10.16967/rpe.v5n1a6

RECIBIDO: 17 de noviembre de 2017 APROBADO: 31 de enero de 2018

CORRESPONDENCIA:

Carlos Eduardo López-Hernández, Calzada Circunvalación Poniente No. 49 Ciudad Granja CP 45010 Zapopan, Jalisco, México.

a Doctor en Dirección de las organizaciones, Profesor de tiempo completo en la Universidad Panamericana Campus Guadalajara, México. Correo electrónico: calopez@up.edu.mx 


\section{¿CÓMO CITO EL ARTÍCULO?} HOW TO CITE THIS PAPER?

CHICAGO:

López-Hernández, Carlos Eduardo. 2018. "Historia del clúster de calzado en Jalisco y su impacto en la estructura industrial". Perspectiva Empresarial 5(1): 69-77. http:// dx.doi.org/10.16967/rpe. v5n1a6

MLA: López-Hernández, Carlos Eduardo. "Historia del clúster de calzado en Jalisco y su impacto en la estructura industrial". Perspectiva Empresarial 5.1 (2018): 6977. Digital. http://dx.doi. org/10.16967/rpe.v5n1a6

\section{History of the Footwear Cluster in Jalisco and its Impact on the Industrial Structure}

ABSTRACT The history of the footwear industry in Mexico shows that its decline is due to many variables, such as the opening of international markets and labor costs, but also the industrial-type organizational structure such as cluster strengthening. In Mexico, like other countries such as the United States, England, China and Brazil, the footwear industry is located and concentrated in some geographical regions, so that the strengthening of the existing relationships among cluster participants is what develops the industry and makes it grow. This research paper describes the industrial structure of the footwear cluster in Jalisco and the historical transformations it has undergone.

KEYWORDS cluster, footwear, large companies, growth.

\section{História do cluster de calçado em Jalisco (México) e o seu impacto na estrutura industrial}

RESUMO A história da indústria do calçado no México demonstra que o seu declive obedece a muitas variáveis, como, por exemplo, a abertura dos mercados internacionais e os custos de mão de obra, mas também a estrutura organizacional de tipo industrial, como é o fortalecimento do cluster. No México, assim como em outros países, como os Estados Unidos, a Inglaterra, a China e o Brasil, a indústria do calçado está localizada e concentrada em algumas regiões geográficas, de maneira que o fortalecimento das relações existentes entre os participantes do cluster e o que desenvolve e faz crescer a indústria. Neste trabalho de pesquisa descreve-se a estrutura industrial do cluster de calçado em Jalisco e as transformações históricas que ele sofreu.

PALAVRAS CHAVE cluster, calçado, grandes empresas e crescimento. 


\section{Introducción}

La decadencia de la shoe industry no solo se da en México, también —de acuerdo con Conklin (1965) - en otros países, como es el caso de Estados Unidos, donde lo han sufrido muchas empresas que tienen más de 25 años de existencia, cuentan con maquinaria inclusive de 20 años de antigüedad y hoy tienen una pobre perspectiva de futuro. La concentración industrial que se presenta en Guadalajara en la industria del calzado ha crecido en cuanto al tamaño de los participantes; sin embargo, algunas de las variables existentes funcionan aún de manera similar a como funcionaban en la época colonial. La apertura comercial de México a mercados internacionales es el principal elemento de la pérdida de competitividad de esta industria a nivel mundial, independientemente de "different disputes regarding the ethics of work e. g. children in countries like China and Brazil" (French \& Wokutch, 2005).

En la segunda parte de esta investigación se estudian las teorías más importantes de Coase y Chandler desde la perspectiva de la teoría de las organizaciones. En este apartado revisaremos la interiorización de la estructura industrial en la estructura organizacional de las empresas. En el tercer apartado, se analiza la teoría de clúster industrial de Porter, ya que nos proporciona el sustento académico necesario para describir gráficamente el funcionamiento de un clúster en cuanto a los participantes y sus relaciones entre ellos.

En la cuarta parte de este trabajo se presenta la evidencia empírica basada en el estudio del clúster industrial del calzado en el estado de Jalisco, México; procedimos con un estudio general de la historia de la industria del calzado en México desde la colonia, y un estudio también del panorama general como parte de la economía nacional y, por último, analizamos el funcionamiento organizacional de la industria del calzado. En el apartado cinco proporcionamos algunas conclusiones.

\section{Historia de la industria del calzado en México}

Esta industria llegó a ser muy importante en México y en Jalisco antes del periodo de sustitución de importaciones. Sin embargo, hacia la mitad de la década de los ochenta sufrió una gran caída (Hernández-Águila, 2006). En 1985 se producían 235,6 millones de pares de zapatos al año; diez años después la producción bajó a 170 millones. La industria del calzado demostró ser una de las industrias más vulnerables a la apertura comercial, en especial de países asiáticos, lo que se suma a la grave crisis económica de la década de los ochenta (Hernández-Águila, 2006). Las micro y pequeñas empresas de esta industria cerraron paulatinamente en este periodo (Iglesias, 1998). Existen autores que mencionan cómo la industria del calzado en México no solo representa una regionalización espacial, sino que este concepto también se corresponde con el tipo de calzado que produce (Iglesias, 1998).

En 1933, en León, el 35 \% de la población económicamente activa se dedicaba a estas manufacturas: existían 12 talleres grandes, 200 medianos y 800 pequeños que agrupaban 10000 empleados, quienes vendían su fuerza de trabajo a las fábricas (Iglesias, 1998). En León, y de manera similar en Guadalajara, se desarrollaron de manera simultánea a diferentes unidades productivas: las grandes y medianas empresas se articularon con talleres domésticos sustentados como unidades productivas de tipo familiar, de manera que estas pequeñas organizaciones industriales fueron la base del desarrollo de esta industria entre 1920 y 1930 (Iglesias, 1998).

Hoover (1933) estudia,

The location of the shoe industry in the specific case of United States and his conclusions are principally the cost of transportation of the finished product, the importance of labor costs in shoe manufacturing, third the cheapening of transportation, forth skill workers and fifth the shoe are an article of drees which must not only fit the individual but the faxhionable.

A nivel mundial los costos de transporte han disminuido en las últimas décadas (Krugman, 1991b), por lo que el abaratamiento mencionado por Hoover se repite en el decaimiento de la industria del calzado en México

Los talleres de calzado han significado no solo unidades de producción que apoyan a los fabricantes o comercializadores, sino también una fuente de mano de obra calificada.

La industria del calzado en México es una industria espacial y sectorialmente zonificada. Ya en la época colonial existían determinados "nódulos espaciales" muy significativos y de ahí surgen los primeros descendientes de maestros zapateros. La Ciudad de México fue el primer lugar. Los maestros y sus 
aprendices eran los únicos que podían vender su producción por pertenecer a estos gremios, aunque lo hacían por medio de un intermediario. De esta forma cubrían casi todo el mercado interno a finales del siglo XVII. (Iglesias, 1998).

Esta forma de organización sectorial y de nódulos que menciona Iglesias (1998) también funcionó en otros países de América, como, por ejemplo, en los Estados Unidos, donde hasta antes de 1852, "shoes had been made by hand in small workshops organized through a putting out system" (Mulligan, 1981, pp. 59).

La organización del trabajo en las unidades domésticas familiares trajo importantes avances en cuanto al acceso directo al mercado por parte de estos productores. Poco a poco florece una multiplicación de untidades productivas de pequeña y gran escalas en donde los capitales locales actúan como responsables principales para que a partir de entonces aquellos espacios se convirtieran en las principales regiones de la industria del calzado nacional. (Iglesias, 1998).

Este tipo de organización de la industria del calzado que menciona Iglesias (1998) no es exclusiva de México, también se repite en otros países como Estados Unidos. Hoover (1933, pp. 259) comenta cómo entre 1760 y 1860 comienza, "the initial specialization, consisting in the setting-up of the individual handicraftsman in the place of family manufacture".

Iglesias (1998) también asegura que con la entrada al GATT ${ }^{1}$ en 1986 México se comprometió a modificar su estructura arancelaria pensando en aprovechar el gran mercado de Estados Unidos, sin embargo, de 1988 a 1989 la industria decrece en poco más de un $18 \%$. A nivel mundial y en otros países esto no sucede; por ejemplo, en 1988 Indonesia abre sus fronteras a capitalistas coreanos y, aunque en ese momento no dominaban el mercado internacional de la industria del calzado, se estableció la Bata Shoe Organization, una de las fábricas más grandes del mundo, cuya producción en ese entonces era equivalente a la de México.

La situación actual de la industria del calzado es diferente y menos competitiva que la de hace 30 años. La apertura de fronteras ha favorecido la entrada de competidores asiáticos, lo cual en gran medida ha afectado el desarrollo de las empresas de calzado no solo en Jalisco sino en todo México.

1 Acuerdo General sobre Aranceles Aduaneros y Comercio.
La Canaical ${ }^{2}$ menciona que la industria del calzado en México genera 266000 trabajos. El $63 \%$ se localiza en Guanajuato, el $16 \%$ en Jalisco —que es donde enfocaremos nuestro estudio-, el $11 \%$ en el estado de México y el $10 \%$ se reparte en todo el país. En el 2008 se importó cerca del $19 \%$, de lo cual el $60 \%$ corresponde a China. El consumo per cápita anual de zapatos se encuentra en 2,7 pares.

Por otra parte, de acuerdo con la Encuesta Industrial Anual de Inegi (2008), aunque el sector manufacturero ha tenido un crecimiento en los últimos años, el sector de fabricación de calzado no ha crecido en la misma proporción.

Unger (2003) desarrolló un diagrama muy detallado del clúster de calzado en León, Guanajuato. Es claro que existen instituciones como las universidades, las financieras y las de comercialización que se encuentran fuera de la organización industrial. Por otra parte, tanto la empresa pequeña como la empresa integrada se ven afectadas por otros jugadores que forman parte del clúster. Los proveedores de transportes, los ganaderos, los proveedores de hule, los curtidores y los proveedores de tela son los principales proveedores que afectan directamente a las empresas tanto pequeñas como integradas.

\section{Funcionamiento del clúster como estructura industrial}

Coase (1937) estudia en su obra The nature of the firm que la principal razón por la que es rentable establecer una firma es porque existe un costo de establecer el mecanismo de precio del mercado. De acuerdo con la afirmación de Coase, el que una empresa dependa del funcionamiento de los trabajadores que no estén dentro de su organización hace menos eficiente a la empresa, caso similar al de algunas empresas de calzado que dependen de pequeños talleres para estar en capacidad de producir. La diferencia también radica en el tamaño de la firma, de manera que Coase (1937) estudia cómo la diferencia entre un tamaño y otro está en el número de transacciones que tiene cada una.

A través de su análisis histórico Chandler probó que las empresas son el factor importante del crecimiento y desarrollo de un país. Asimismo, cómo su surgimiento y crecimiento solo podría

\footnotetext{
2 Cámara Nacional de la Industria del Calzado (Canaical).
} 
llevarse por medio de una jerarquía interna. Sus estudios se centran en el cambio industrial y la interrelación de las grandes corporaciones; esto lo explica en su libro La mano visible: la revolución administrativa en las empresas americanas (1977).

Para las empresas que llegan primero y desarrollan su "base de aprendizaje", esta corresponde al mismo tiempo a una barrera para los próximos entrantes. El interés de Chandler (1962; 1990) en las grandes firmas no es porque sean los villanos o salvadores, sino porque funcionan como "fuerzas creativas" que afectan la economía y, desafortunadamente, la mayoría de las personas no lo reconocen.

En Strategy and structure: chapters in the history of the american industrial enterprise (1962) describe cuatro grandes firmas: General Motors, Standard Oil, DuPont y Sears. En las cuatro, Chandler argumenta que la estructura multi-divisional tanto de línea como de staff no era una copia arbitraria de la estructura militar, sino una respuesta natural a la complejidad de los negocios.

Otra de sus obras - The visible hand (1977) describe cómo hace un siglo los consumidores repentinamente se encontraron con artículos más baratos y suficientes para sus necesidades.

Uno de sus últimos libros, Scale and scope: the dynamics of industrial capitalist (1990), propone que la evolución se presenta en el capitalismo y no tanto en América. Chandler ha llegado a afirmar que si los Estados Unidos creen que van a lograr competir globalmente con pequeñas empresas están equivocados.

Un clúster industrial es un grupo de firmas en la misma localización que componen un sistema de producción con derramas que pueden ser verticales u horizontales (Madsen \& Smith, 2003). Uno de los autores de inicios del siglo pasado que más influyó en autores modernos como Krugman y Porter fue Alfred Marshall. Las ideas de Alfred Marshall (1920) de hace casi un siglo tienen un impacto mayúsculo en nuestra era, pues autores tan influyentes como Krugman (1991, pp. 484485) aseguran haber tomado como base los hallazgos de este economista.

Marshall $(1920 ; 1935)$ identifica fuerzas que conducen a que la industria se localice en regiones específicas de un país y explica también las ventajas de las empresas que se concentran en un espacio. Estas ventajas se conocen como economías externas y son: 1. La disponibilidad de proveedores de insumos especializados tanto en variedad como en costos más económicos; 2 . Que exista mano de obra suficiente y calificada en la industria de referencia; y 3. Que la información se difunda rápidamente (gracias a la cercanía entre las industrias la información fluye de manera más efectiva).

Para Porter (1998) los clústeres son concentraciones geográficas de compañías e instituciones interconectadas en una actividad particular, y pueden incluir a proveedores de materias primas y maquinaria, prestadores de servicio, canales de comercialización y clientes, e inclusive lateralmente a empresas de la competencia; asimismo, el Gobierno y otras instituciones tales como universidades, asociaciones y proveedores de información.

Porter (1998) menciona que las compañías que forman parte de un clúster pueden ser inclusive más productivas con relación a aquellas que no forman parte de uno. Esta productividad es posible gracias a cinco factores: 1 . Un mejor acceso a empleados y proveedores (e. g. es más probable que recluten empleados experimentados); 2. Acceso a información especializada (e. g. las condiciones propias del clúster hacen que la información sea más fácil de transferir); 3 . Complementariedades (e. g. en el clúster del turismo la satisfacción del visitante no solo es gracias a la principal atracción del lugar, sino a los complementadores como hoteles, restaurantes, transportes, etc.); 4 . Acceso a instituciones y bienes públicos (e. g. formación de laboratorios de prueba o diseño dependientes del clúster); y 5. Mejor motivación y medición (e. g. la empresa conoce bien los precios de los proveedores y los empleados saben dónde otorgan las mejores condiciones de trabajo).

De acuerdo con estos cinco factores cada miembro del clúster obtiene beneficios como si fuera más grande o como si se hubiera fusionado con otra empresa, pero sin sacrificar flexibilidad. De acuerdo con Porter (1998), la innovación además de la productividad es otro de los beneficios que obtienen los miembros del clúster. El aprendizaje entre las compañías se presenta más fácilmente gracias a su acercamiento y trato caracara. También pueden incurrir en bajos costos en el desarrollo de nueva tecnología o de desarrollos innovadores.

Unger, Detraux, Martínez \& Revilla (2003) aseguran que una vez un clúster inicia su formación existe un ciclo de autoreforzamiento que promueve su propio crecimiento, especialmente cuando las instituciones locales lo apoyan y la competencia es vigorosa. Los clústeres constantemente 
evolucionan cuando surgen y desaparecen empresas en su interior, o cuando cambian las instituciones que las soportan. La cercanía entre los proveedores y los usuarios generan fortalezas competitivas (Unger et al., 2003).

Estas fuerzas de aglomeración surgen del clúster, entre los clústeres de esa industria y entre clústeres relacionados con ella (Delgado, Porter \& Stern, 2011).

La teoría de los clústeres industriales nos ayuda a entender la relación con diversos actores externos por parte de la empresa, de modo que también revisamos cómo estas interacciones tienen afectaciones al interior de la organización. En la siguiente etapa de este trabajo presentaremos la evidencia empírica apoyada del caso del clúster de calzado en Guadalajara.

\section{Clúster de calzado en Jalisco}

Se ha revisado cómo a ciertas empresas les beneficia la cercanía a mercados, a proveedores de materias primas e inclusive de mano de obra calificada. Este es el caso del clúster de calzado en Guadalajara. Primero, se revisan los antecedentes históricos de la industria; en segundo lugar, se realiza una descripción general de la industria a nivel nacional, así como del clúster más importante de calzado en el país — que es el de Guanajuato-; y tercero, se analiza el clúster de calzado en Guadalajara y se propone un modelo de su organización.

\section{Clúster de calzado en Guanajuato}

Históricamente, la industria del calzado se ha aglomerado en ciertas regiones geográficas como en el caso de Estados Unidos (Hoover, 1933; Mulligan, 1981), Inglaterra (Rydberg, 1965), y sin duda China y Brazil (French \& Wokutch, 2005) en la ciudad de Franca en el estado de Sao Paulo-. México no ha sido la excepción.

Las conclusiones presentadas por el Seijal (2009) coinciden con el análisis de Unger et al. (2003) sobre los clústeres ${ }^{3}$ industriales en México.

3 "Redes de firmas interdependientes, instituciones de producción del conocimiento que conectan tanto a las instituciones como a los clientes en una cadena de producción agregada de valor". (OECD, 1998, p. 43, como se cita en Unger et al., 2003).
Unger ha sido uno de los autores más influyentes en el tema de clúster y de especialización regional en México, e inclusive tomamos como base el diagrama del clúster de calzado en Guanajuato realizado por él. Afirma que se ha dado un aprendizaje de la zona de León (y Guadalajara) gracias al producto de una larga experiencia, lo cual ha generado una concentración de la producción nacional en esta región.

\section{Clúster de calzado en Jalisco}

En la Encuesta de Coyuntura de la Industria del Calzado de Jalisco (2004) encontramos a actores de la industria del calzado como proveedores de materias primas, mano de obra, servicios, comercialización, inmuebles e instalaciones, transportes y administrativos, además de empresas de financiamiento, capacitación (universidades) y apoyo al comercio exterior.

Para algunos autores -Madsen \& Smith (2003), por ejemplo- en países como Dinamarca los municipios se toman como unidades geográficos para definir los clústeres y deben contar por lo menos con 10 firmas. Para estos dos autores la ventaja de una firma, al pertenecer a un clúster de otras firmas similares a nivel horizontal, es que logra mejorar su productividad en casi un $8 \%$.

Se realizaron diferentes entrevistas a profundidad y los datos de contacto los proporcionó la Cámara de la Industria del Calzado en Jalisco (Cecij). Se logró concretar una propuesta de la organización y el funcionamiento del clúster de la industria del calzado en Jalisco. Esta primera aproximación presenta la interacción de la estructura externa de la empresa con la estructura organizacional e interna de la empresa.

Las materias primas que se utilizan en la fabricación de calzado son principalmente piel, avíos (suelas, tacones y plantas), así como accesorios (hebillas) y empaque. Existen proveedores de ciertos materiales indirectos como, por ejemplo, químicos. La producción de zapatos la realizan fabricantes (tienen su propia marca) o maquiladores (no tienen marca), quienes surten a los comercializadores y los fabricantes.

La existencia de talleres continúa. Son microempresas que realizan trabajos por "encargo" de los fabricantes o de los maquiladores, y su labor, principalmente, consiste en ayudar a completar ciertos trabajos que superan la capacidad instalada de los productores. 

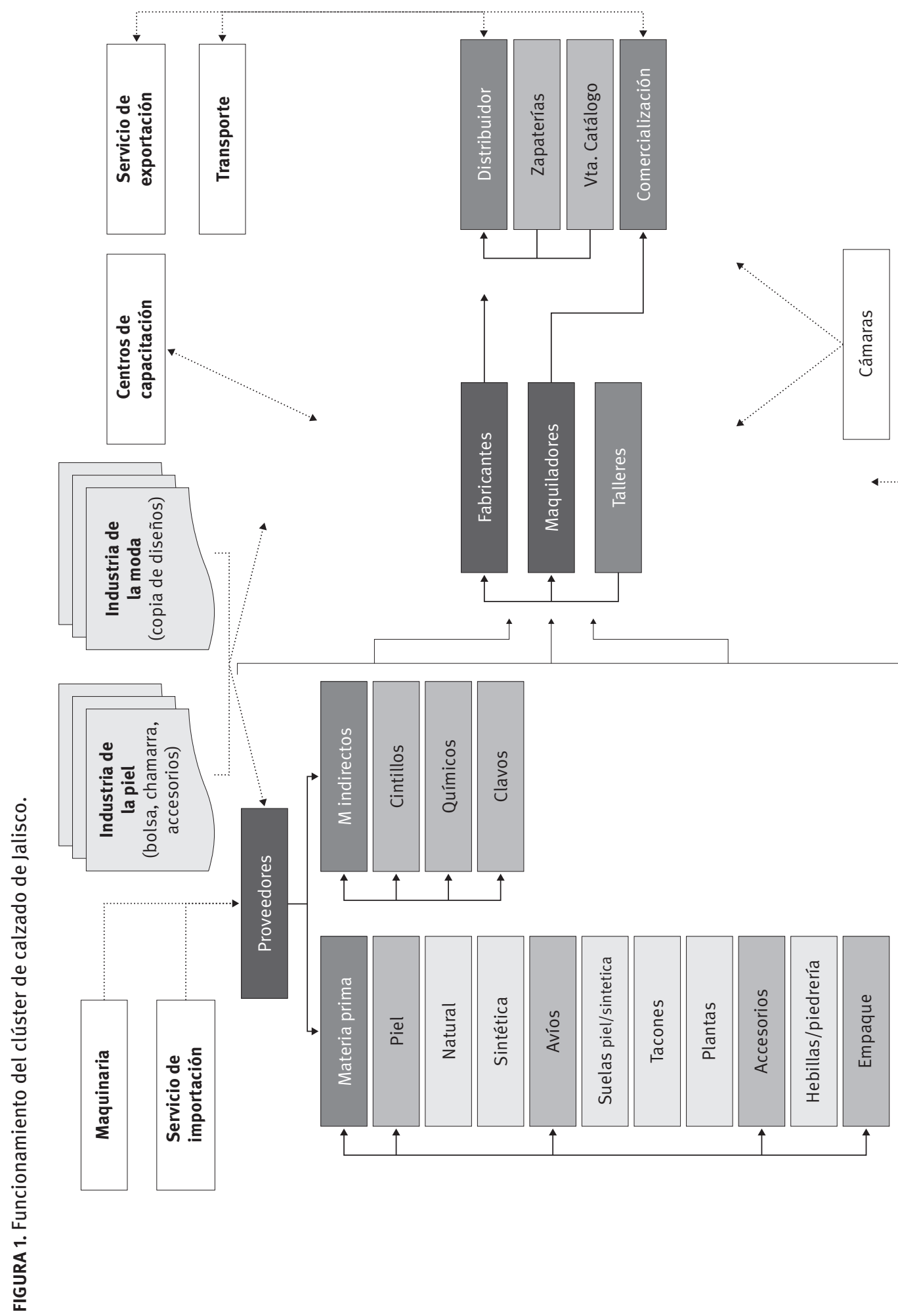
El calzado se puede comercializar en el mercado nacional o de exportación. Existen también distribuidores, quienes manejan varias marcas; el mercado tradicional -el de las zapateríasquienes compran directamente de los fabricantes y distribuidores; otro canal de venta no convencional ha sido el del catálogo, el cual, en los últimos años ha crecido. Los comercializadores son dueños de la marca pero no producen, por eso requieren de los maquiladores y los talleres para producir sus zapatos.

La industria de la piel y de la moda son entidades que afectan directamente el funcionamiento del clúster. En el caso de la piel su impacto es sobre las materias primas $\mathrm{y}$, por tanto, incide en la calidad del producto ya terminado. De acuerdo con Unger et al. (2003) las actividades entre la industria de la piel y del calzado están muy integradas, pero han sufrido un rezago en su calidad frente a la piel de importación, lo que pone en riesgo su capacidad integradora.

La industria de la moda afecta a las empresas de esta industria porque es quien genera los nuevos modelos. Año con año la industria de calzado de Jalisco participa en ferias internacionales, en especial en Italia donde revisan las nuevas tendencias en cuanto a nuevos modelos y materiales. Unger (2003) menciona que el Gobierno podría propiciar la formación de diseñadores con el fin de mejorar el desempeño de esta industria.

La maquinaria es considerada una herramienta más en la producción de calzado. Existen de diferentes calidades y es la mejor la importada de Europa (la maquinaria ha sido clave en el desarrollo de esta industria). Hoover (1933) comenta que en el caso americano la introducción de la McKAy sewing machine entre el periodo de 1860 y 1900 redujo el requerimiento de mano de obra. Ciertos servicios como, por ejemplo, el de importación (en especial materias primas y maquinaria), y el de exportación para la comercialización, son importantes en el desarrollo de esta industria. El transporte, tanto local como foráneo, es un actor que afecta directamente el desempeño y la competitividad de la industria del calzado (aunque algunas empresas grandes tienen su propia flotilla, la gran mayoría no cuenta con este transporte). Existen centros de capacitación, aunque los talleres son una fuente de mano de obra calificada para las empresas más grandes, así como también las mismas empresas cumplen con esta función.

Por último, la influencia de la cámara ha sido importante en propiciar la convergencia de los intereses de los distintos participantes. En Jalisco los industriales del calzado, representados por la Cámara de la Industria del Calzado del estado de Jalisco, organizaban muestras de calzado en 1978 (Cecij, 1978). Además, en sus reuniones revisaban temas de mejora común, como, por ejemplo, políticas de compra de materias prima y política de ventas, así como cursos de capacitación (modelado de calzado), con el fin de hacer frente a la falta de personal. Los efectos de las derramas a través de economías relacionadas es fundamental para el crecimiento y la creación de empleo en un amplio rango de industrias y regiones; estas derramas pueden inclusive traspasar fronteras geográficas (Delgado, Porter \& Stern, 2011).

\section{Conclusiones}

En este trabajo encontramos que las empresas de calzado del clúster en Guadalajara, Jalisco, no se integran a cierto nivel, sino prefieren dejar ciertas partes de su negocio a otras entidades dentro del clúster. Por ejemplo, los talleres suplen en algunas ocasiones la capacidad de producción que les falta a las fábricas o maquiladoras, así como existen empresas que comercializan pero no producen. La capacitación del personal en ocasiones no se realiza en la empresa, sino en los talleres, o al cambiarse el empleado de firma. La parte del diseño, en general, se deja a cargo de la industria de la moda; los transportes y los servicios de importación y exportación en una gran mayoría de empresas se dejan a terceros. Se prueba, por tanto, que la estructura interna de la empresa depende de su entorno externo y este se afecta de diversas maneras.

Se ha logrado demostrar que la organización y la estructura interna de las empresas que pertenecen a este clúster extienden su estructura más allá de sus fronteras organizacionales, de manera que revisaremos también qué afectaciones sufre la estructura y la organización de la empresa por variables que interactúan en el clúster al que pertenecen, pero que no forman parte de ella. Aunque las empresas de calzado en Jalisco cuentan con una larga tradición en la producción no encontramos algún ejemplo significativamente similar al caso de Bata Shoe Organization. Se propone, entonces, que en futuras investigaciones se profundice en este caso particular de estudio a fin de responder por qué creció y por qué ha permanecido tantos años en el mercado, y tratar de definir 
las variables y las diferencias de esta empresa con el propósito de realizar propuestas de mejora a las empresas que forman parte del clúster de calzado en Jalisco, México.

\section{REFERENCIAS}

BataShoe Mexico. (2011). Bata México. Recuperado de http://www.batamexico.com.mx/about-us/heritage.html

Cámara de la Industria del Calzado de Jalisco. (2004). Encuesta de Coyuntura Industria del Calzado 2004. Guadalajara: Sistema Estatal de Información Jalisco.

Cecij. (1978, agosto 18). Muestra del calzado. El Informador.

Chandler, A. (1962). Strategy and structure: chapters in the history of the american industrial enterprise. Cambridge, MA: MIT Press.

Chandler, A. D. (1977). The visible hand. Cambridge, MA: Harvard University Press.

Chandler, A. D. (1990). Scale and scope: the dynamics of industrial capitalist. Cambridge, MA: Harvard University Press.

Coase, R. (1937, noviembre). The nature of the firm. Economica, New Series, 386-405.

Conklin, H. (1965). The awakening of a sleeping giant: the shoe industry. Financial Analysts Journal, 59-64.

Delgado, M., Porter, M. \& Stern, S. (2011). Clusters, convergence, and economic performance (Sometido a revisión para publicación). Harvard Business School. Institute for Strategy and Competitiveness, 1-49.

French, J. \& Wokutch, R. (2005). Child Workers, Globalization, and international business ethics: a case study in brazil's export-oriented shoe industry. Business Ethics Quarterly, 615-640.

Greiner, L. E. (1998). Evolution and revolution as organizations grow. Harvard Business Review Classic, 1-11.

Hernández-Águila, E. D. (2006). Estrategias de reestructuración productiva de los empresarios tapatíos de la industria del calzado. Guadalajara, Jalisco: Universidad de Guadalajara.
Hoover, E. (1933). The location of the shoe industry in the United States. The Quarterly Journal of Economics, 47(2), 254-276.

Iglesias, E. (1998). La industria del cuero y del calzado en México. México D. F.: Instituto de Investigaciones Económicas, UNAM.

Inegi. (2008). Encuesta industrial anual. Ciudad de México D. F.

Kleiner, A. (2002). Professor Chandler's Revolution. Strategy+Business, 1-8.

Krugman, P. (1991b). Increasing returns and economic geography. The Journal of Political Economy, 483499.

Krugman, P. (1991). Increasing returns and economic geography. The Journal of Political Economy, 483499.

Krugman, P. (1991). Increasing returns and economic geography. The Journal of Political Economy, 483499.

Madsen, E. \& Smith, V. (2003). Industrial clusters, firm location and productivity. Department of Economics Aarhus School of Business, 1-16.

Marshall, A. (1920). Principles of economics. Nueva York: Cosimo Inc.

Marshall, A. (1935). Pure theory of foreign trade. Londres: The London School of Economics and Political Science.

Mulligan, W. (1981). Mechanization and work in the american shoe industry: Lynn, Massachusetts 1852-1883. The Journal of Economic History, 41(1), 59-63.

Porter, M. E. (1998, noviembre-diciembre). Clusters and the new economics of competition. Harvard Business Review, 77-90.

Rydberg, H. (1965). The location of the english shoe industry. Geografiska Annaler. Series B, Human Geography, 47(1), 44-55.

Sistema Estatal de Información Jalisco-Seijal. (2009, primer semestre). Encuesta de coyuntura industria del calzado. Jalisco, Mexico.

Unger, K., Detraux, B., Martínez, A. \& Revilla, M. (2003). Los clústeres industriales en México: especializaciones regionales y la política Industrial. Santiago de Chile: División de Desarrollo Productivo y Empresarial de la Cepal. 
